\begin{tabular}{|c|c|}
\hline Title & $\begin{array}{l}\text { Effects of statins on thrombosis development in patients with sy stemic lupus erythematosus and antiphospholipid } \\
\text { antibodies }\end{array}$ \\
\hline Author(s) & $\begin{array}{l}\text { Watanabe, T .; Oku, K.; A mengual, O.; Hisada, R.; Ohmura, K.; Nakagawa, I.; Shida, H.; Bohgaki, T .; Horita, T.; } \\
\text { Y asuda, S.; A tsumi, T. }\end{array}$ \\
\hline Citation & $\begin{array}{l}\text { Lupus, 27(2), 225-234 } \\
\text { https://doi.org/10.1177/0961203317716787 }\end{array}$ \\
\hline Issue Date & $2018-02$ \\
\hline Doc URL & http:/hdl.handle.net/2115/68460 \\
\hline Rights & $\begin{array}{l}\text { T W atanabe, K Oku, O A mengual, R Hisada, K Ohmura, I Nakagawa, H Shida, T Bohgaki, T Horita, S Y asuda, T } \\
\text { A tsumi, Effects of statins on thrombosis development in patients with systemic lupus erythematosus and } \\
\text { antiphospholipid antibodies, L upus, V ol 27, I ssue 2, 225-234. Copyright @ } 2018 \text { The A uthor(s). Reprinted by } \\
\text { permission of SA GE Publications. }\end{array}$ \\
\hline Tyре & article (author version) \\
\hline File Information & Lupus27_225.pdf \\
\hline
\end{tabular}

Instructions for use 


\title{
Effects of Statins on Thrombosis Development in Patients with Systemic Lupus Erythematosus and Antiphospholipid Antibodies
}

\author{
Toshiyuki Watanabe ${ }^{1}$, Kenji Oku ${ }^{1}$, Olga Amengual ${ }^{1}$, Ryo Hisada ${ }^{1}$, Kazumasa Ohmura ${ }^{1}$, \\ Ikuma Nakagawa ${ }^{1}$, Haruki Shida ${ }^{1}$, Toshiyuki Bohgaki ${ }^{1}$, Tetsuya Horita ${ }^{1}$, Shinsuke Yasuda ${ }^{1}$, \\ and Tatsuya Atsumi ${ }^{1}$ \\ ${ }^{1}$ Division of Rheumatology, Endocrinology and Nephrology, Hokkaido University Graduate \\ School of Medicine, Sapporo, Japan
}

Short title: statin effects for thrombosis in SLE with aPL

Correspondence to:

Tatsuya Atsumi, MD, PhD

Division of Rheumatology, Endocrinology and Nephrology,

Hokkaido University Graduate School of Medicine

N15W7, Kita-Ku Sapporo, Hokkaido, Japan 060-8638

E-mail: at3tat@med.hokudai.ac.jp 


\begin{abstract}
To identify the effects of statins and risk factors for thrombosis in patients with newly onset of systemic lupus erythematosus (SLE) with or without antiphospholipid antibodies (aPL). Consecutive patients with SLE without history of thrombotic events were retrospectively enrolled from April, 1997 to February, 2014. The development of first thrombosis and death caused by thrombosis were defined as the study endpoint. Risk and protective factors for developing thrombosis were analyzed. One hundred and fifty-two patients, 80 positive and 72 negative for aPL, were included. In aPL positive patients, 15 developed arterial (n = 6) and venous $(\mathrm{n}=9)$ thrombosis (median follow-up period 69 months). Cox's proportional hazards model showed that older age at SLE onset and IgG-anticardiolipin antibodies (aCL) were statistically significant risks for thrombosis. Statin therapy was identified as a statistically significant protective factor against thrombosis (hazard ratio 0.12, 95\% confidence interval 0.01-0.98). In aPL negative patients (median follow-up period 46 months), 7 patients developed thrombosis (5 arterial and 2 venous). No risk factors for thrombosis were found in this group. In aPL positive patients with SLE, the late disease onset and the presence of IgG-aCL represented additional risk factors for thrombosis. Statin treatment appeared as a protective factor for thrombosis.
\end{abstract}


Keywords. Systemic Lupus Erythematosus, Antiphospholipid Antibodies, Thrombosis, Statins 


\section{INTRODUCTION}

Systemic lupus erythematosus (SLE) is an inflammatory multisystem autoimmune disease characterized by the production of multiple autoantibodies and immune complexes, causing severe multi-organ damage including nephritis and central nervous system disorder. The prognosis of SLE has been improved with 5-year survival rates over 95\% presumably due to the advance of effective immunosuppressive therapy and management against infectious complications (1). Consequently, the major cause of death in SLE has turned into cardiovascular diseases (CVD) and thrombosis (2). Nowadays, thrombosis is one of the most frequent complications in patients with SLE, significantly affecting the morbidity and mortality (3).

A number of risk factors for thrombosis in SLE patients have been identified. Among them, the presence of antiphospholipid antibodies (aPL), detected up to a half of SLE patients, is a well-documented risk for the occurrence of thrombosis (4-6). Antiphospholipid antibody is a heterogeneous family of autoantibodies with different specificities including lupus anticoagulant (LA), anticardiolipin antibodies (aCL), anti- $\beta 2$-glycoprotein I antibodies (aß2GPI) and phosphatidylserine dependent antiprothrombin antibodies (aPS/PT) $(7,8)$. The occurrence of thrombotic events, as well as obstetric complications, in association with the persistent presence of aPL is defined as antiphospholipid syndrome (APS).

Apart from aPL, a number of other risk factors for thrombosis, such as older age at SLE onset, male sex, high disease activity, obesity, have been reported by some cohort studies $(4,9,10)$. On the other hand, few data are available regarding the primary prevention of thrombosis, although many studies focused in the prevention of recurrent 
thrombotic events (11-15).

Statins, the 3-hydroxy-3-methylglutaryl coenzyme A reductase inhibitors, are frequently prescribed for SLE patients to treat dyslipidemia, primary and/or secondary to the use of corticosteroids. These drugs have been proven to be effective also for primary and secondary prevention of CVD and venous thrombosis in some large studies (16-19). Based on the in vitro effect of statins on endothelial cells activated by aPL, statins may have some advantages in patients with APS (20-22), but other clinical studies have failed to show their benefit (23).

We assumed that the mechanisms and risk factors of thrombosis would be different between SLE patients with and without aPL. The main purpose of this study was to investigate the effects of statins on thrombosis prevention in SLE patients with or without aPL. Moreover, we investigated the risk or protective factors for thromboembolic complications in a cohort of SLE patients 


\section{PATIENTS AND METHODS}

\section{Patients}

Newly diagnosed patients with SLE attending at our Rheumatology clinic from April, 1997 to February, 2014 were consecutively included in this retrospective cohort study. SLE was diagnosed according to the 1997 American College of Rheumatology (ACR) revised criteria for SLE $(24,25)$. Patients with a history of thrombosis and those who had received any anti-thrombotic agent prior to or at the time of SLE diagnosis were excluded.

\section{Study design}

The primary outcome was to identify the development of the first thrombotic event after inclusion. At the first visit to our clinic, all patients underwent physical and laboratory examination by certified rheumatologists and the diagnosis of SLE was established by ACR criteria $(24,25)$. Patients were followed-up at our clinic up to June 2014. The occurrences of the first thrombotic events or deaths during the follow-up period were recorded. Arterial thrombosis was defined as cerebral infarction (CI), acute coronary syndrome, and other internal organ arterial thrombosis such as intestinal infarction, renal infarction. Venous thrombosis comprised deep vein thrombosis (DVT) or pulmonary embolism (PE). Thrombosis should be symptomatic and confirmed by at least one of the following methods: doppler ultrasound, computed tomographic (CT) scanning, magnetic resonance imaging (MRI), nuclear medicine scan, or histopathology. Brain CT and brain MRI were performed in SLE patients with symptoms suspected of a cerebrovascular attack such as dysarthria, paralysis, disturbance of consciousness, and visual disorders. Haemorrhagic and thrombotic 
stroke could be distinguished by the different enhancements patterns on brain CT and brain MRI.

To evaluate risk and protective factors, medical records were retrospectively reviewed and the following demographic, clinical, and laboratory data were verified by the authors: age at disease onset, gender, family history of thrombosis, malar rash, discoid rash, photosensitivity, oral ulcers, arthritis, serositis, lupus nephritis (LN), neurological disorder, haemolytic anemia, leukopenia (white blood cell $<4,000 / \mathrm{mm}^{3}$ or lymphocyte $<1,500 / \mathrm{mm}^{3}$ ), thrombocytopenia (platelets $<100,000 / \mathrm{mm}^{3}$ ), C3, C4, CH50, anti DNA antibodies, LA, IgG/IgM-aCL, IgG/IgM-aß2GPI, IgG/IgM-aPS/PT. Disease activity was evaluated by Systemic Lupus Erythematosus Disease Activity Index 2000 (SLEDAI-2K) (26). Historical profile of traditional risk factors for thrombosis was evaluated: smoking history, obesity (body mass index $>25 \mathrm{~kg} / \mathrm{m}^{2}$ ), oral contraceptives, hypertension (systolic blood pressure > $140 \mathrm{~mm} \mathrm{Hg}$, diastolic blood pressure $>90 \mathrm{~mm} \mathrm{Hg}$, or use of antihypertensive drugs at the time of SLE diagnosis), dyslipidemia (total cholesterol > $220 \mathrm{mg} / \mathrm{dl}$, fasting triglycerides > $150 \mathrm{mg} / \mathrm{dl}$, high density lipoprotein cholesterol $<40 \mathrm{mg} / \mathrm{dl}$, or using lipid lowering drugs at the time of SLE diagnosis), diabetes mellitus (haemoglobin A1c $>6.5 \%$ at baseline or using antidiabetic drugs at the time of SLE diagnosis).

The initiation of new drugs within three months from SLE onset was also analyzed. In our hospital, the specific treatment for newly diagnosed SLE patients is established during the hospitalization period extended up to 3 months. Therefore, we have included in the analysis the use of medications started within three months from SLE onset. These drugs included corticosteroids, immunosuppressive agents (cyclophosphamide, cyclosporine, tacrolimus, mycophenolate mofetil, azathioprine, methotrexate and mizoribine), 
anti-thrombotic agents (low dose aspirin, ticlopidine, clopidogrel, dipyridamole, cilostazol, warfarin and heparin), statins and vitamin D (alfacalcidol, calcitriol and eldecalcitol). All of these drugs were prescribed according to the clinicians' judgment.

\section{Detection of aPL}

LA was detected according to the previous version of guidelines recommended by the Subcommittee on Lupus Anticoagulant/ Antiphospholipid Antibody of the Scientific and Standardization Committee of the International Society on Thrombosis and Haemostasis (27). For measurement of the activated Partial Thromboplastin Time, a sensitive reagent with low phospholipid concentration (test PTT-LA; Diagnostica Stago) was used for screening and mixing test, and the results were confirmed with the use of a Staclot LA kit (Diagnostica Stago). The dilute Russell's viper venom time was screened and confirmed by use of a Gradipore LA test (Sydney New South Wales, Australia). The kaolin clotting time was measured using a kaolin solution (Dade Behring, Liederbach, Germany) with the standard protocol. The cut-off levels for the LA tests were previously established as $>$ 99th percentile of 40 healthy subjects, as our routine laboratory assays (28). Testing for IgG/IgM-aCL was performed using the standard aCL enzyme-linked immunosorbent assay (ELISA) (29). IgG/IgM-aß2GPI were determined by ELISA, as previously reported (30). IgG/IgM-aPS/PT were measured by in-house ELISAs, as previously described (7). Patients were defined as aPL positive if at least one of the tests described above was positive.

The study was performed in accordance with the Declaration of Helsinki and the Principles of Good Clinical Practice. Approval was obtained from local ethics committee. 


\section{Statistical analysis}

Continuous variables were expressed as the mean \pm standard deviation (SD) if normally distributed, or as the median and quartiles if they had a skewed distribution. Categorical variables were described as counts and percentages. Fisher's exact test, Student's t test or Mann-Whitney U test were carried out appropriately. Kaplan-Meier curves were applied to evaluate the time to first development of thrombosis and event-free survival. Log-rank test was used to compare Kaplan-Meier curves associated with thrombosis. P values less than 0.05 were considered as statistically significant.

In multivariate analysis, variables that had statistical significance $(\mathrm{p}<0.05)$ in log-rank test were included. Age and sex were entered in multivariate model, regardless of statistical significance in the univariate analysis. After excluding variables that had strong association with the other variables by Pearson's test, the risk and protective factors for thrombosis were analyzed by multivariate Cox's proportional hazards model. All statistical analyses were performed using SPSS software, version 22.0 (Chicago, Illinois, USA). 


\section{RESULTS}

A total of one hundred and fifty-two Japanese patients (138 female, 14 male) without previous history of thrombotic events nor use of anti-thrombotic agents were enrolled in the study. Eighty patients had aPL and 72 patients were negative for any aPL tested at baseline. Table 1 shows the baseline characteristics of all patients. Thirteen out of 51 patients positive for LA had aPS/PT (11 had IgG, 5 IgM and 3 both IgG/IgM). The median age was 30 (interquartile range [IQR] 22-41) years and 33 (IQR 24-46) years in aPL positive and negative groups, respectively. The median follow-up periods for aPL positive and negative groups were 69 (IQR 27-118) months and 46 (IQR 25-105), respectively.

As for clinical and laboratory features associated with SLE, such as mucocutaneous disorders, haematological abnormalities, serositis, and neurological involvement, no statistical significances were observed between aPL positive and negative patients.

Regarding traditional risk for thrombosis, the prevalence of arterial hypertension was significantly higher in patients negative for aPL (25\%) compared with those positive for aPL $(10 \%)(p=0.018)$. There were no statistically significant differences in the prevalence of other traditional risk factors between the two groups.

The analysis of treatment started within three months after SLE onset showed that aPL positive patients were less frequently treated with corticosteroids and immunosuppressive agents than those without aPL $(\mathrm{p}=0.018, \mathrm{p}=0.013$, respectively). No statistical differences were found in the usage of statin or anti-thrombotic agents between two 
groups.

The median follow-up period in the whole cohort of SLE patients was 65 (IQR 28-117) months. Twenty-two (14\%) patients developed thrombosis; 15 (19\%) in aPL positive group and 7 (9.7\%) in aPL negative group. Nine (11\%) patients with aPL vs. two (2.8\%) patients without aPL had venous thrombosis $(\mathrm{p}=0.059$, log-rank test), and six $(7.5 \%)$ patients with aPL vs. five (6.9\%) patients without aPL had arterial thrombosis $(p=0.998)$. In aPL positive group, CI was observed in 6 (7.5\%) patients, DVT alone in 4 (5.0\%), PE alone in 3 (3.8\%), and both DVT and PE at the same time in 2 (2.5\%). In aPL negative group, CI was found in 4 (5.6\%) patients, intestinal infarction in $1(1.4 \%)$, DVT alone in 1 (1.4\%) and PE alone in 1 (1.4\%). The median periods for the occurrence of thrombosis after SLE diagnosis were 6.0 (IQR 1.5-63) months for aPL positive group and 2.0 (IQR 0.5-10) months for aPL negative group.

Thirteen thrombotic events occurred within one year from the diagnosis of SLE. Among patients with those early thrombosis, 8 events occurred in aPL positive group and 5 in aPL negative group. Furthermore, nine (aPL positive in 5 and negative in 4) and thirteen patients (aPL positive in 10 and negative in 3) developed thrombosis within and after three months from SLE onset, respectively. The incidence rate of thrombosis in aPL positive and negative group was 2.92 per 100 patient-years and 1.78 per 100 patient-years, respectively. During the observational period, thirty four patients (22\%) were lost for follow-up. The median follow-up period was 42 (IQR 12-87) months. Eight patients were lost within the $1^{\text {st }}$ year from the enrollment and 15 patients were lost from $1^{\text {st }}$ to $5^{\text {th }}$ years of follow-up. Further, 3 out of the 34 patients died due to infection, liver failure and suicide at 2, 8 and 18 months after starting follow-up, respectively. There was no death related to thrombosis. 
Table 2 shows the characteristics of patients divided in four groups, with or without thrombosis and aPL positive or negative during the follow-up period. Results of log-rank analysis in the four groups are shown in Table 3. When analyzing the clinical manifestations in aPL positive group, log-rank analysis revealed that aPL positive patients who developed thrombosis had a lower frequency of $\operatorname{LN}(n=3,20 \%)$ than those without thrombosis $(\mathrm{n}=32,49 \%)(\mathrm{p}=0.024)$. In the aPL negative group, log-rank test indicated that age at SLE onset (every ten age) was significantly increased in the thrombosis group ( $\mathrm{p}=$ 0.002). The frequency of hypertension was significantly higher in patients with thrombosis (4/7: $57 \%)$ than in those without $(14 / 65: 22 \%)(p=0.034)$. The relationship between aPL profiles and thrombosis was also analyzed. IgG-aCL positive was found in 5 out of 15 (33\%) patients who developed thrombosis but only in 7 out of 65 (11\%) patients without thrombosis $(\mathrm{p}=0.003)$. The frequency of IgG-aß2GPI was higher in patients with thrombosis $(p=0.043)$. The other aPL evaluated in this study (LA, IgM-aCL, IgM-aß2GPI, IgG/IgM-aPS/PT) were not identified as risk factors for thrombosis. The use of corticosteroids, immunosuppressive agents, anti-thrombotic drugs or vitamin $\mathrm{D}$, within 3 months from the disease onset, was not related to the occurrence of thrombosis in SLE patients with or without aPL.

In this study, 46 patients received statins during three months following the diagnosis of SLE. The relationship between subsequent thrombotic events and statin therapy is shown in Figure 1. In aPL positive patients, 1 out of 15 (6.7\%) patients with thrombosis and 22 out of 65 (34\%) patients without thrombosis received statins. The patient with aPL who developed thrombosis on statin therapy had both DVT and PE. Atorvastatin was administered in 13 patients, fluvastatin in 4 , simvastatin in 4 and rosvastatin in 2 . The 
median duration of statin therapy was 30 (IQR 5.0-40) months. Statin treatment was found to be significantly protective against the development of thrombosis $(p=0.024)$. In aPL positive patients with statins, the level of total cholesterol, low density lipoprotein (LDL)-cholesterol and the frequency of hypertension and LN were higher compared to those without statins $(\mathrm{p}=0.003, \mathrm{p}=0.011, \mathrm{p}=0.040$ and $\mathrm{p}=0.024$, respectively) (Table 4). In aPL negative patients, 2 (29\%) patients with thrombosis and 21 (32\%) without thrombosis received statin treatment. No relationship was observed between statin treatment and thrombosis prevention.

The results of univariate analysis by log-rank test indicated that IgG-aCL and IgG-a $\beta 2$ GPI are candidate risk factors for thrombosis. LN and statin treatment are likely to be protective for developing thrombosis. Many additional prothrombotic factors might be involved in the development of thrombosis in patients with LN, such as hypertension, dyslipidemia, anticoagulant drugs, and statin therapy. In this cohort, there was a strong association between LN and statin usage $(|\mathrm{r}|=0.275$, $\mathrm{p}=0.014)$ and between IgG-aCL and IgG-aß2GPI $(|\mathrm{r}|=0.364, \mathrm{p}=0.001)$. Therefore, we excluded LN and IgG-aß2GPI as covariates in the multivariate model.

Including gender and age as variables, multivariate analysis with Cox’s proportional hazards model showed that older age at SLE onset and IgG-aCL were statistically significant risk for thrombosis (hazard ratio (HR) 1.83 for every ten age, 95\% confidence interval (C.I.) 1.12-2.99 and HR 7.18, 95\%C.I. 1.82-28.4, respectively) (Figure 2). Moreover, statin therapy played a protective role in developing thrombosis (HR 0.12, 95\%C.I. 0.01-0.98). In patients without aPL, age at SLE onset, hypertension and gender were included in multivariate analysis. None of these risk factors was correlated with the development of 
thrombosis.

Analysis according to the criteria aPL

Currently, aPS/PT are frequently found in APS patients but are not yet included in the APS laboratory criteria. We have also analyzed the efficacy of statins in aPL positive patients defined according to the criteria aPL, and therefore excluding aPS/PT.

Seventy-six out of 152 SLE patients had aPL according to APS criteria (8). During 69 (IQR 27-118) months, 14 patients developed thrombosis. Log-rank analysis revealed that the frequency of LN was lower in patients with thrombosis $(\mathrm{p}=0.017)$, and that the frequencies of IgG-aCL positive and IgG-a $\beta 2 \mathrm{GPI}$ positive were higher in thrombosis group ( $p=0.001, p=0.036$, respectively). Statin treatment significantly reduced the incidence of thrombosis $(\mathrm{p}=0.041)$ (supplementary figure 1). However, multivariate analysis indicated that statin therapy did not prevent from developing thrombosis (HR 0.16, 95\%C.I. 0.02-1.30). 


\section{DISCUSSION}

In the present study, we showed that older age at SLE onset and IgG-aCL are risk for subsequent thrombosis in newly diagnosed SLE patients with aPL. More importantly, we found that the initiation of statins within three months of SLE onset reduced the risk of developing thrombosis, suggesting statins as potential candidate for primary prophylaxis in early SLE patients positive for aPL.

The relationship between older age at SLE onset and thrombotic risk has been examined in large cohort studies. LUMINA (LUpus in MInorities: NAture vs nurture) study, a multiethnic longitudinal study of outcome, demonstrated that older age at disease onset was a risk factor for arterial and venous thrombosis $(9,31)$. Moreover, Kaiser et al., confirmed these findings in a very large cohort of 1930 SLE patients (32). The present study supports the published evidences demonstrating that older age at SLE onset represents a risk for thrombosis development.

It has been already well recognized that aPL increases the risk for thrombosis in SLE (32-34). In our study, the incidence rate of thrombosis in SLE patients with and without aPL was $19 \%$ and $9.7 \%$, respectively. Similar results were observed in another cohort study 
reported by Tektonidou et al., in which thrombosis rate was $20.1 \%$ for aPL positive and $7.6 \%$ for aPL negative (10). We focused on the presence of aPL rather than on the APS diagnosis and included aPS/PT in the definition of aPL positive. Although aPS/PT have not been included in the APS criteria yet, several reports showed that aPS/PT are highly prevalent in APS patients and strongly correlate with LA and thrombosis (7). Regarding aPL profile related to thrombosis, presence of LA or aCL in SLE patients elevates the risk for arterial and venous thrombosis $(10,32,34,35)$. In concordance with previous reports, our study showed that IgG-aCL are associated with thrombosis. On the other hand, we could find neither LA nor aPS/PT as a thrombotic risk in our cohort. LA was more prevalent in our newly diagnosed SLE patients compared with other studies. SLE patients have massive polyclonal B cell activation with production of multiple autoantibodies at onset, and standard corticosteroid/immunosuppressive therapy downregulate such activation of autoantibody production. LA comprises heterogeneous population of aPL with major and minor anticoagulant functions, thus a number of non-specific LA can be found in new and active SLE patients before immunosuppression. In fact, 64\% of our patients had LA, more frequent than more specific aPL such as aCL, a $32 \mathrm{GPI}$ or aPS/PT. We do not intend to exclude the role of LA in clinical practice, but such characteristic of LA in immunologically very active SLE patients should be clarified in the next future. The frequency of aPS/PT in our thrombotic patients tended to be higher than in non-thrombotic, although the difference was not statistically significant. It may be due to the study sample size.

The present study shows that thrombosis tends to occur within the first year of disease regardless of the presence or absence of aPL. Previous reports indicated that the incidence of thrombosis increased in the first year following SLE diagnosis (36, 37). 
Plausible explanations of early thrombosis may include high disease activity, inflammatory states and the initiation of corticosteroid treatment $(9,36,38)$.

The frequency of venous thrombosis appeared to be higher in aPL positive group than in aPL negative group. However, the incidence of arterial or venous thrombosis in each group was too small to conduct statistical subgroup analyses.

Although thrombosis is a common and critical manifestation in SLE patients with aPL, few studies have addressed the primary prophylaxis for thrombosis. Some reports described that low dose aspirin reduced the risk of thrombosis in SLE patients with aPL (10, 39). In contrast, a prospective study, reported by Erkan et al., showed that administration of low dose-aspirin for aPL positive individuals without a history of thrombosis, including SLE patients, was not beneficial for primary prevention of thrombosis (40). Therefore, the protective effect of low dose aspirin as primary prophylaxis for thrombosis is still controversial. Other studies reported that hydroxychloroquine had a beneficial role for primary prophylaxis $(10,41)$. However, limited studies have shown the primary anti-thrombotic effect in clinical practice of SLE, although hydroxychloroquine is routinely used in SLE patients (23).

In our study, statin treatment was demonstrated to be effective to decrease the risk of developing thrombotic events in SLE patients with aPL. The main pharmacological effect of statins is to lower serum cholesterol levels, reducing atherosclerosis and CVD events resulted from atherosclerosis. The "pleiotropic effects" of statins have been recognized (19, 42) and some clinical studies successfully revealed their anti-thrombotic effects (43-46). Although some works elucidated the preventive effects of statin therapy in SLE-related atherosclerosis, a meta-analysis of randomized controlled trial showed that statins could not 
improve the atherosclerosis in SLE patients (47). Our study revealed that statins could reduce the occurrence of thrombosis in patients with aPL but not in those without aPL, indicating that statins might have other anti-thrombotic mechanisms in SLE patients with aPL and not related to the prevention of atherosclerosis. A number of experimental studies have shown that statins downregulate the most fundamental procoagulant function of aPL. Statins reduced production of tissue factor (TF), intracellular adhesion molecule-1 (ICAM-1) and interleukin-6 induced by aPL in human umbilical vein endothelial cells $(20,21)$. An in vivo study showed that treatment with fluvastatin of APS model mice decreased the size of thrombosis and soluble ICAM-1 level (48). Lopez-Pedrera et al. demonstrated the in vivo effect of statins in reducing procoagulant molecule expression (22). Another clinical study strengthened the evidence of statin effects for aPL positive patients using biomarkers of thrombin generation (49). Although statin treatment reduces aPL-induced procoagulant factors both in vitro and in vivo, clinical benefits have not been shown in any study so far. A task force reports at the 14th international congress on aPL concluded as follows; it is difficult to establish the evidence of statin treatment for primary prevention of thrombosis because the large number of patients and the long-term follow-up time will be necessary to find statistically significant differences between current treatment and new statin therapeutic approaches (23).

Vitamin D deficiency and insufficiency are related to thrombotic manifestation in APS. The administration of vitamin $\mathrm{D}$ has been expected to decrease the development of thrombosis (23). Contrary to our expectation, this study could not verify the anti-thrombotic effect of vitamin D in SLE patients. The lack of anti-thrombotic effect of vitamin D in our cohort may be explained by the small number of patients included in the study. 
Our study certainly had some limitations. This is a single-center based retrospective observational study, all patients are Japanese, and the number of patients is small so that the results might not be generalized. Additionally, biomarkers associated with thrombosis were not available and the mechanisms of protective effect of statin for thrombosis are obscure. Other limitation is that the decision for the statin usage was based on the clinical judgment of the treating physicians, which might produce a selective bias. Further, the significance on statin treatment is marginal, especially when considering the upper limit of the C.I. (HR 0.12, 95\%C.I. 0.01-0.98). The marginally significant difference observed in statin usage between aPL positive patients with and without thrombosis might be affected by the small number of patients in each group because multivariate analysis can be easily influenced by the sample size. One of the major limitations is that due to its retrospective design the statin treated patients were different from the rest in several aspects. In aPL positive patients treated with statins, the level of total cholesterol, LDL-cholesterol and the prevalence of hypertension and LN were statistically higher than in those without statins (Table 4). However, long-term observation based on detailed clinical records was possible in this study design, leading to a new evidence.

To our knowledge, this study is the first report to demonstrate that statins potentially prevents SLE patients with aPL from developing thrombosis. We believe that our study have given a clue to consider primary prophylaxis for thrombosis by statins. Prospective multicenter studies including large numbers of patients will confirm the beneficial role of statins.

In conclusion, this study confirmed that the late SLE onset and the presence of IgG-aCL represent additional risk factors for thrombosis in SLE patients with aPL. 
Furthermore, our data suggest that statins at early phase of aPL positive SLE patients have a potential role against developing thrombosis.

\section{ACKNOWLEDGEMENTS}

We thank Ms. Mayumi Shitamichi for technical assistance and Dr. Yuichiro Fujieda, Dr.

Takashi Kurita and Dr. Michihito Kono for statistical analysis and helpful suggestions.

Funding: The work was supported by a grant by the Japanese Ministry of Health, Labor, and Welfare and by the Japanese Ministry of Education, Culture, Sports, Science, and Technology.

Disclosure statement: Dr. Tatsuya Atsumi has received honoraria and/or research funding from Mitsubishi Tanabe Pharma Co. and Pfizer Inc. All other authors have declared no conflicts of interest. 


\section{REFERENCES}

1. Feldman CH, Hiraki LT, Winkelmayer WC, et al. Serious infections among adult Medicaid beneficiaries with systemic lupus erythematosus and lupus nephritis. Arthritis Rheumatol 2015; 67: 1577-1585.

2. Cervera R, Khamashta MA, Font J, et al. Morbidity and mortality in systemic lupus erythematosus during a 10-year period: a comparison of early and late manifestations in a cohort of 1,000 patients. Medicine (Baltimore) 2003; 82: 299-308.

3. Bernatsky S, Boivin JF, Joseph L, et al. Mortality in systemic lupus erythematosus. Arthritis Rheum 2006; 54: 2550-2557.

4. Mok CC, Tang SS, To CH, Petri M. Incidence and risk factors of thromboembolism in systemic lupus erythematosus: a comparison of three ethnic groups. Arthritis Rheum 2005; 52: $2774-2782$.

5. Love PE, Santoro SA. Antiphospholipid antibodies: anticardiolipin and the lupus 
anticoagulant in systemic lupus erythematosus (SLE) and in non-SLE disorders. Prevalence and clinical significance. Ann Intern Med 1990; 112: 682-698.

6. Harris EN, Gharavi AE, Asherson RA, Boey ML, Hughes GR. Cerebral infarction in systemic lupus: association with anticardiolipin antibodies. Clin Exp Rheumatol 1984; 2: 47-51.

7. Atsumi T, Ieko M, Bertolaccini ML, et al. Association of autoantibodies against the phosphatidylserine-prothrombin complex with manifestations of the antiphospholipid syndrome and with the presence of lupus anticoagulant. Arthritis Rheum 2000; 43: 1982-1993.

8. Miyakis S, Lockshin MD, Atsumi T, et al. International consensus statement on an update of the classification criteria for definite antiphospholipid syndrome (APS). J Thromb Haemost 2006; 4: 295-306.

9. Calvo-Alen J, Toloza SM, Fernandez M, et al. Systemic lupus erythematosus in a multiethnic US cohort (LUMINA). XXV. Smoking, older age, disease activity, lupus anticoagulant, and glucocorticoid dose as risk factors for the occurrence of venous thrombosis in lupus patients. Arthritis Rheum 2005; 52: 2060-2068.

10. Tektonidou MG, Laskari K, Panagiotakos DB, Moutsopoulos HM. Risk factors for thrombosis and primary thrombosis prevention in patients with systemic lupus erythematosus with or without antiphospholipid antibodies. Arthritis Rheum 2009; 61: 29-36.

11. Crowther MA, Ginsberg JS, Julian J, et al. A comparison of two intensities of warfarin for the prevention of recurrent thrombosis in patients with the antiphospholipid antibody syndrome. N Engl J Med 2003; 349: 1133-1138.

12. Finazzi G, Marchioli R, Brancaccio V, et al. A randomized clinical trial of 
high-intensity warfarin vs. conventional antithrombotic therapy for the prevention of recurrent thrombosis in patients with the antiphospholipid syndrome (WAPS). J Thromb Haemost 2005; 3: 848-853.

13. Garcia DA, Khamashta MA, Crowther MA. How we diagnose and treat thrombotic manifestations of the antiphospholipid syndrome: a case-based review. Blood 2007; 110: $3122-3127$.

14. Lim W, Crowther MA, Eikelboom JW. Management of antiphospholipid antibody syndrome: a systematic review. JAMA 2006; 295: 1050-1057.

15. Ruiz-Irastorza G, Hunt BJ, Khamashta MA. A systematic review of secondary thromboprophylaxis in patients with antiphospholipid antibodies. Arthritis Rheum 2007; 57: 1487-1495.

16. Ridker PM, Group JS. Rosuvastatin in the primary prevention of cardiovascular disease among patients with low levels of low-density lipoprotein cholesterol and elevated high-sensitivity C-reactive protein: rationale and design of the JUPITER trial. Circulation 2003; 108: 2292-2297.

17. Biere-Rafi S, Hutten BA, Squizzato A, et al. Statin treatment and the risk of recurrent pulmonary embolism. Eur Heart J 2013; 34: 1800-1806.

18. Prevention of cardiovascular events and death with pravastatin in patients with coronary heart disease and a broad range of initial cholesterol levels. The Long-Term Intervention with Pravastatin in Ischaemic Disease (LIPID) Study Group. N Engl J Med 1998; 339: 1349-1357.

19. Glynn RJ, Danielson E, Fonseca FA, et al. A randomized trial of rosuvastatin in the prevention of venous thromboembolism. N Engl J Med 2009; 360: 1851-1861. 
20. Meroni PL, Raschi E, Testoni C, et al. Statins prevent endothelial cell activation induced by antiphospholipid (anti-beta2-glycoprotein I) antibodies: effect on the proadhesive and proinflammatory phenotype. Arthritis Rheum 2001; 44: 2870-2878.

21. Ferrara DE, Swerlick R, Casper K, et al. Fluvastatin inhibits up-regulation of tissue factor expression by antiphospholipid antibodies on endothelial cells. J Thromb Haemost 2004; 2: 1558-1563.

22. Lopez-Pedrera C, Ruiz-Limon P, Aguirre MA, et al. Global effects of fluvastatin on the prothrombotic status of patients with antiphospholipid syndrome. Ann Rheum Dis 2011; 70: 675-682.

23. Erkan D, Aguiar CL, Andrade D, et al. 14th International Congress on Antiphospholipid Antibodies Task Force Report on Antiphospholipid Syndrome Treatment Trends. Autoimmun Rev 2014; 13: 685-696.

24. Hochberg MC. Updating the American College of Rheumatology revised criteria for the classification of systemic lupus erythematosus. Arthritis Rheum 1997; 40: 1725.

25. Tan EM, Cohen AS, Fries JF, et al. The 1982 revised criteria for the classification of systemic lupus erythematosus. Arthritis Rheum 1982; 25: 1271-1277.

26. Gladman DD, Ibanez D, Urowitz MB. Systemic lupus erythematosus disease activity index 2000. J Rheumatol 2002; 29: 288-291.

27. Brandt JT, Triplett DA, Alving B, Scharrer I. Criteria for the diagnosis of lupus anticoagulants: an update. On behalf of the Subcommittee on Lupus Anticoagulant/Antiphospholipid Antibody of the Scientific and Standardisation Committee of the ISTH. Thromb Haemost 1995; 74: 1185-1190.

28. Pengo V, Tripodi A, Reber G, et al. Update of the guidelines for lupus anticoagulant 
detection. Subcommittee on Lupus Anticoagulant/Antiphospholipid Antibody of the Scientific and Standardisation Committee of the International Society on Thrombosis and Haemostasis. J Thromb Haemost 2009; 7: 1737-1740.

29. Harris EN, Gharavi AE, Patel SP, Hughes GR. Evaluation of the anti-cardiolipin antibody test: report of an international workshop held 4 April 1986. Clin Exp Immunol 1987; 68: 215-222.

30. Amengual O, Atsumi T, Khamashta MA, Koike T, Hughes GR. Specificity of ELISA for antibody to beta 2-glycoprotein I in patients with antiphospholipid syndrome. $\mathrm{Br} \mathrm{J}$ Rheumatol 1996; 35: 1239-1243.

31. Toloza SM, Uribe AG, McGwin G, JR., et al. Systemic lupus erythematosus in a multiethnic US cohort (LUMINA). XXIII. Baseline predictors of vascular events. Arthritis Rheum 2004; 50: 3947-3957.

32. Kaiser R, Cleveland CM, Criswell LA. Risk and protective factors for thrombosis in systemic lupus erythematosus: results from a large, multi-ethnic cohort. Ann Rheum Dis 2009; 68: 238-241.

33. Cervera R, Khamashta MA, Font J, Sebastiani GD, Gil A, Lavilla P, et al. Morbidity and mortality in systemic lupus erythematosus during a 5-year period. A multicenter prospective study of 1,000 patients. European Working Party on Systemic Lupus Erythematosus. Medicine (Baltimore) 1999; 78: 167-175.

34. Wahl DG, Guillemin F, de Maistre E, Perret C, Lecompte T, Thibaut G. Risk for venous thrombosis related to antiphospholipid antibodies in systemic lupus erythematosus--a meta-analysis. Lupus 1997; 6: 467-473.

35. Finazzi G, Brancaccio V, Moia M, et al. Natural history and risk factors for 
thrombosis in 360 patients with antiphospholipid antibodies: a four-year prospective study from the Italian Registry. Am J Med 1996; 100: 530-536.

36. Sarabi ZS, Chang E, Bobba R, et al. Incidence rates of arterial and venous thrombosis after diagnosis of systemic lupus erythematosus. Arthritis Rheum 2005; 53: 609-612.

37. Romero-Diaz J, Garcia-Sosa I, Sanchez-Guerrero J. Thrombosis in systemic lupus erythematosus and other autoimmune diseases of recent onset. J Rheumatol 2009; 36: 68-75.

38. Johannesdottir SA, Horvath-Puho E, Dekkers OM, et al. Use of glucocorticoids and risk of venous thromboembolism: a nationwide population-based case-control study. JAMA Intern Med 2013; 173: 743-752.

39. Wahl DG, Bounameaux H, de Moerloose P, Sarasin FP. Prophylactic antithrombotic therapy for patients with systemic lupus erythematosus with or without antiphospholipid antibodies: do the benefits outweigh the risks? A decision analysis. Arch Intern Med 2000; 160: 2042-2048.

40. Erkan D, Harrison MJ, Levy R, et al. Aspirin for primary thrombosis prevention in the antiphospholipid syndrome: a randomized, double-blind, placebo-controlled trial in asymptomatic antiphospholipid antibody-positive individuals. Arthritis Rheum 2007; 56: 2382-2391.

41. Ho KT, Ahn CW, Alarcon GS, et al. Systemic lupus erythematosus in a multiethnic cohort (LUMINA): XXVIII. Factors predictive of thrombotic events. Rheumatology (Oxford) 2005; 44: 1303-1307.

42. Kwak B, Mulhaupt F, Myit S, Mach F. Statins as a newly recognized type of immunomodulator. Nat Med 2000; 6: 1399-1402. 
43. Tsai AW, Cushman M, Rosamond WD, et al. Coagulation factors, inflammation markers, and venous thromboembolism: the longitudinal investigation of thromboembolism etiology (LITE). Am J Med 2002; 113: 636-642.

44. Undas A, Brummel-Ziedins KE, Mann KG. Statins and blood coagulation. Arterioscler Thromb Vasc Biol 2005; 25: 287-294.

45. Sen-Banerjee S, Mir S, Lin Z, et al. Kruppel-like factor 2 as a novel mediator of statin effects in endothelial cells. Circulation 2005; 112: 720-726.

46. Kaba NK, Francis CW, Moss AJ, et al. Effects of lipids and lipid-lowering therapy on hemostatic factors in patients with myocardial infarction. J Thromb Haemost 2004; 2: $718-725$

47. Ye Y, Zhao X, Xie H, Tian Z, Zhang S. Efficacy and safety of statins in the prevention of atherosclerosis in patients with systemic lupus erythematosus--a meta-analysis of randomized controlled trials. Int J Cardiol 2013; 167: 301-303.

48. Ferrara DE, Liu X, Espinola RG, et al. Inhibition of the thrombogenic and inflammatory properties of antiphospholipid antibodies by fluvastatin in an in vivo animal model. Arthritis Rheum 2003; 48: 3272-3279.

49. Erkan D, Willis R, Murthy VL, et al. A prospective open-label pilot study of fluvastatin on proinflammatory and prothrombotic biomarkers in antiphospholipid antibody positive patients. Ann Rheum Dis 2014; 73: 1176-1180. 
Table 1 Characteristics and laboratory data of the patients

$$
\text { aPL positive }(n=80) \quad \text { aPL negative }(n=72) \quad \text { P value }
$$

\section{Characteristics}

Female sex, n (\%)

Age, median [IQR] (years)

Family history of thrombosis, n (\%)

SLEDAI-2K, mean \pm SD

Traditional risk for thrombosis
72 (90)

$30 \quad[22-41]$

7 (8.8)

$14.6 \pm 7.2$

$15.5 \pm 8.5$

0.494 
Smoking, n (\%)

Obesity (body mass index > 25), n (\%)

Oral contraceptive drugs, n (\%)

Hypertension, n (\%)

Dyslipidemia, n (\%)

Diabetes, n (\%)

LN, n (\%)

aPL profile

LA, n (\%)

IgG-aCL, n (\%) > 19.2

IgM-aCL, n (\%) > 23.4

IgG-aß2GPI, n (\%) > 2.2

IgM-a $\beta 2$ GPI, $n(\%) \quad>6.0$

IgG-aPS/PT, n (\%) > 2.0

IgM-aPS/PT, n (\%) > 5.2

Initial treatment*

Corticosteroids, n (\%)

Immunosuppressive agents, $\mathrm{n}(\%)$

Statins, n (\%)

Anti-thrombotic agents, n (\%)

Vitamin D
28 (35)

$12(15)$

$0(0)$

$8(10)$

$50(63)$

3 (3.8)

35 (44)

$51(64)$

12 (15)

12 (15)

11(/77) (14)

15(/77) (19)

20 (25)

9 (11)
24 (34)

0.865

9 (13)

0.815

$0(0)$

1.000

18 (25)

0.018

48 (67)

0.615

$2(2.8)$

1.000

31 (43)

1.000

67 (84)

69 (96)

0.018

23 (29)

35 (49)

0.013

23 (29)

23 (32)

0.725

26 (33)

21 (29)

0.726

49 (61)

45 (63)

1.000 
Data are expressed as median [interquartile range: IQR], mean \pm standard deviation (SD) or percentage. $\quad *<3$ months after SLE diagnosis, aPL: antiphospholipid antibodies, n: number, SLEDAI-2K: Systemic Lupus Erythematosus Disease Activity Index 2000, LN: lupus nephritis, LA: lupus anticoagulant, aCL: anticardiolipin antibodies, aß2GPI: anti- $\beta 2$-glycoprotein I antibodies, aPS/PT: phosphatidylserine-dependent antiprothrombin antibodies.

Table 2 Characteristics of patients with and without thrombotic events

$$
\text { aPL positive }(n=80) \quad \text { aPL negative }(n=72)
$$

Thrombosis Non thrombosis Thrombosis Non thrombosis

$$
(\mathrm{n}=15) \quad(\mathrm{n}=65) \quad(\mathrm{n}=7) \quad(\mathrm{n}=65)
$$

Characteristics

$\begin{array}{llccc}\text { Female sex, n (\%) } & 13(87) & 59(91) & 7(100) & 59(91) \\ \text { Age, median [IQR] (years) } & 36[27-48] & 28[20-40] & 54[45-60] & 32[21-45] \\ \text { Family history of thrombosis, n (\%) } & 1(6.7) & 6(9.2) & 0(0) & 2(3.1)\end{array}$


Traditional risk for thrombosis

$$
\text { Smoking, n (\%) }
$$

Obesity (body mass index > 25), n (\%)

Oral contraceptive drugs, n (\%)

Hypertension, n (\%)

Dyslipidemia, n (\%)

Diabetes, n (\%)

LN, n (\%)

aPL profile

$$
\text { LA, n (\%) }
$$

IgG-aCL, n (\%) > 19.2

IgM-aCL, n (\%) > 23.4

IgG-aß2GPI, n (\%) > 2.2

IgM-a $\beta 2$ GPI, n (\%) > 6.0

IgG-aPS/PT, n (\%) > 2.0

IgM-aPS/PT, n (\%) > 5.2

Initial treatment

Corticosteroids, n (\%)

Immunosuppressive agents, n (\%)

Statins, n (\%)

\section{Initial treatin}

5 (33)

23 (35)

2 (29)

$22(34)$

4 (27)

$8(12)$

$1(14)$

$8(12)$

$0(0)$

$0(0)$

$0(0)$

$0(0)$

3 (20)

5 (7.7)

4 (57)

$14(22)$

$12(80)$

38 (58)

6 (86)

$42(65)$

1 (6.7)

2 (3.1)

$0(0)$

2 (2.1)

3 (20)

32 (49)

3 (43)

28 (43)
$10(67)$

5 (33)

4 (27)

5(/15) (33)

2(/15) (13)

7 (47)

2 (13)
7 (11)

$41(73)$

7 (11)

8 (12)

6(/62) (9.7)

13(/62) (21)

13 (20)

7 (11)

$12(80)$

55 (85)

7 (100)

62 (95)

3 (20)

20 (31)

3 (43)

32 (49)

1 (6.7)

22 (34)

2 (29)

21 (32) 
Anti-thrombotic agents, n (\%)

Vitamin D
$6(40)$

$7(47)$
$20(31)$

$42(65)$
$2(29)$

$3(43)$
$19(29)$

$42(65)$

Data are expressed as median [interquartile range: IQR], mean \pm standard deviation (SD) or percentage. aPL: antiphospholipid antibodies, n: number, SLEDAI-2K: Systemic Lupus Erythematosus Disease Activity Index 2000, LN: lupus nephritis, LA: lupus anticoagulant, aCL: anticardiolipin antibodies, aß2GPI: anti- $\beta 2$-glycoprotein I antibodies, aPS/PT: phosphatidylserine-dependent antiprothrombin antibodies.

Table 3 Univariate analysis by log-rank test to select the thrombotic factors

$$
\text { aPL positive }(n=80) \quad \text { aPL negative }(n=72)
$$

P value P value

Age (every ten age)

0.608

0.002

Hypertension

0.243

0.034

LN

0.024

0.999

IgG-aCL

0.003

IgG-a $\beta 2 G P I$

0.043 
aPL: antiphospholipid antibodies, n: number, LN: lupus nephritis, aCL: anticardiolipin antibodies, aß2GPI: anti- $\beta 2$-glycoprotein I antibodies.

Table 4 Characteristics and laboratory data of aPL positive SLE patients treated with or without statins

$$
\text { Statin }+(n=23) \quad \text { Statin }-(n=57) \quad \text { P value }
$$

\section{Characteristics}

Female sex, n (\%)

Age, median [IQR] (years)

Family history of thrombosis, n (\%)

SLEDAI-2K, mean \pm SD
20 (87)

32 [18-43]

$3(13)$

$16.0 \pm 7.2$
$52(91)$

0.683

$29[22-39] \quad 0.836$

$4(7.0)$

0.404

$14.1 \pm 7.2$

0.279 
Traditional risk for thrombosis

Smoking, n (\%)

Obesity (body mass index > 25), n (\%)

Hypertension, n (\%)

Dyslipidemia, n (\%)

Total cholesterol, mg/dl

Triglyceride, mg/dl

HDL-cholesterol, mg/dl

LDL-cholesterol, mg/dl

Diabetes, n (\%)

LN, n (\%)

aPL profile

LA, n (\%)

IgG-aCL, $\mathrm{n}(\%) \quad>19.2$

IgM-aCL, n (\%) > 23.4

IgG-aß2GPI, n (\%) > $>2.2$

IgM-aß2GPI, n (\%) > 6.0

IgG-aPS/PT, n (\%) > $\quad 2.0$

IgM-aPS/PT, n (\%) > 5.2
9 (39)

19 (33)

$9(16)$

1.000

$5(22)$

$3(5.3)$

0.040

14 (61)

36 (63)

1.000

$196.8 \pm 56.0$

$161.2 \pm 44.2$

0.003

$168.3 \pm 76.6$

$142.4 \pm 89.2$

0.226

$51.2 \pm 16.3$

$112.0 \pm 47.9$

1 (4.3)

15 (65)

$46.4 \pm 17.3$

0.255

$86.3 \pm 36.1$

0.011

2 (3.5)

1.000

20 (35)

0.024

37 (65)

0.800

1 (4.3)

11 (19)

0.164

4 (17)

8 (14)

0.736

2 (8.7)

9 (16)

0.497

7 (30)

8 (14)

0.113

3 (13)

17 (30)

0.157

3 (13)

6 (11)

0.712

Data are expressed as median [interquartile range: IQR], mean \pm standard deviation (SD) or percentage. aPL: antiphospholipid antibodies, SLE: systemic lupus erythematosus, n: number, 
SLEDAI-2K: Systemic Lupus Erythematosus Disease Activity Index 2000, HDL: high density lipoprotein, LDL: low density lipoprotein, LN: lupus nephritis, LA: lupus anticoagulant, aCL: anticardiolipin antibodies, aß2GPI: anti- $\beta 2$-glycoprotein I antibodies, aPS/PT: phosphatidylserine-dependent antiprothrombin antibodies. 


\section{FIGURE LEGENDS}

Figure 1 Kaplan-Meier survival curves for thrombosis in patients treated (solid line) and not treated (dashed line) with statins.

A) aPL positive SLE patients $(p=0.024), B)$ aPL negative SLE patients $(p=0.840)$

Figure 2 Multivariate analysis with Cox's proportional hazards model in aPL positive SLE patients.

Higher age at SLE onset and the presence of IgG-aCL were identified as risk factors for thrombosis (Hazard ratio (HR) 1.83 for every ten age, 95\% confidence interval (C.I.) 1.12-2.99 and HR 7.18, 95\%C.I. 1.82-28.4, respectively). Statin treatment was protective for thrombosis onset (HR 0.12, 95\%C.I. 0.01-0.98).

Supplementary Figure 1 Analysis according to criteria aPL: Kaplan-Meier survival curves for thrombosis in patients treated (solid line) and not treated (dashed line) with statins excluding aPS/PT from aPL.

A) aPL positive SLE patients $(p=0.041)$, B $)$ aPL negative SLE patients $(p=0.588)$ 

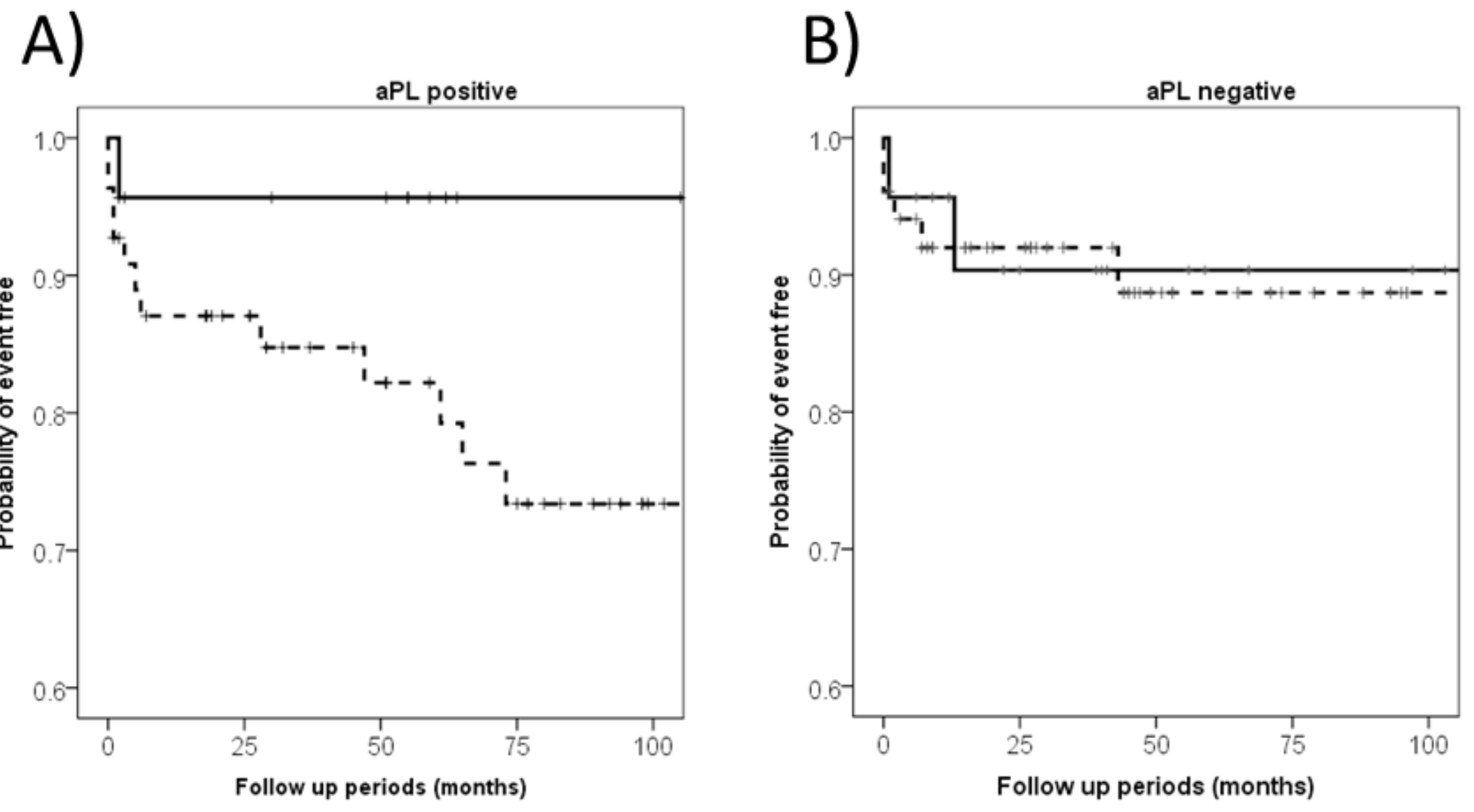


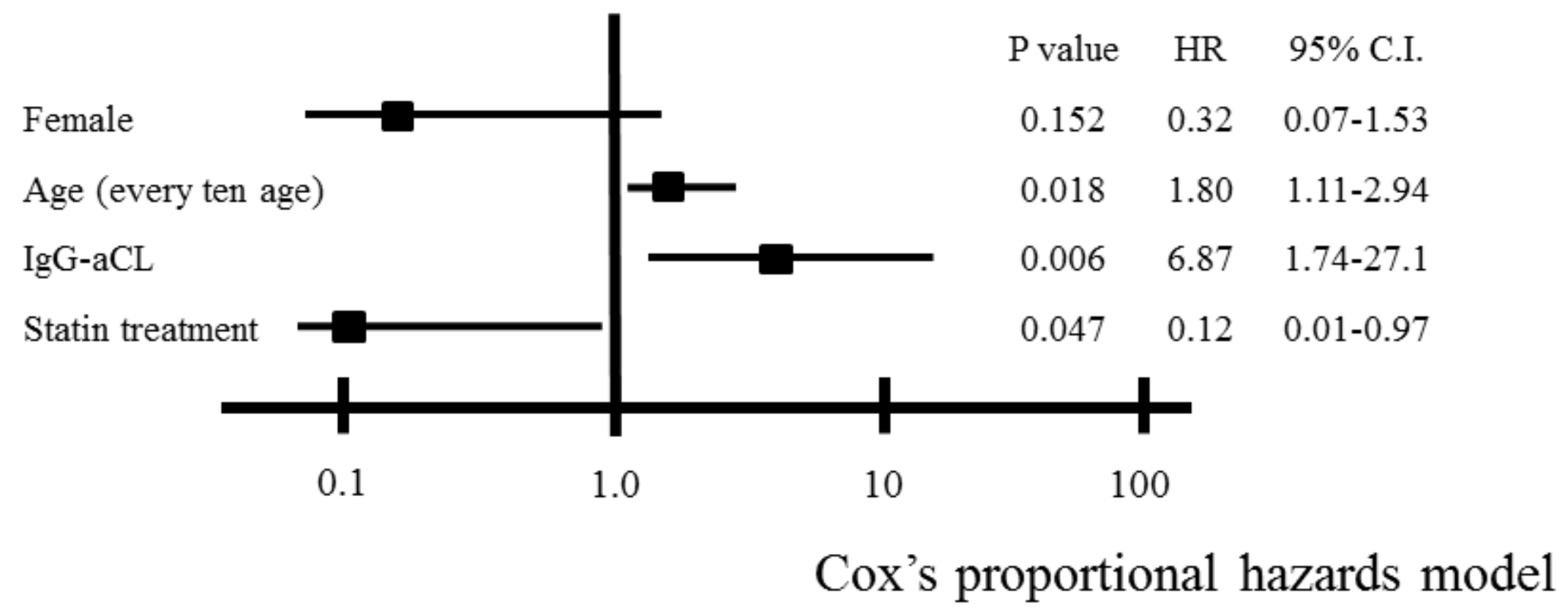



A)
B)

Supplementary Figure 1
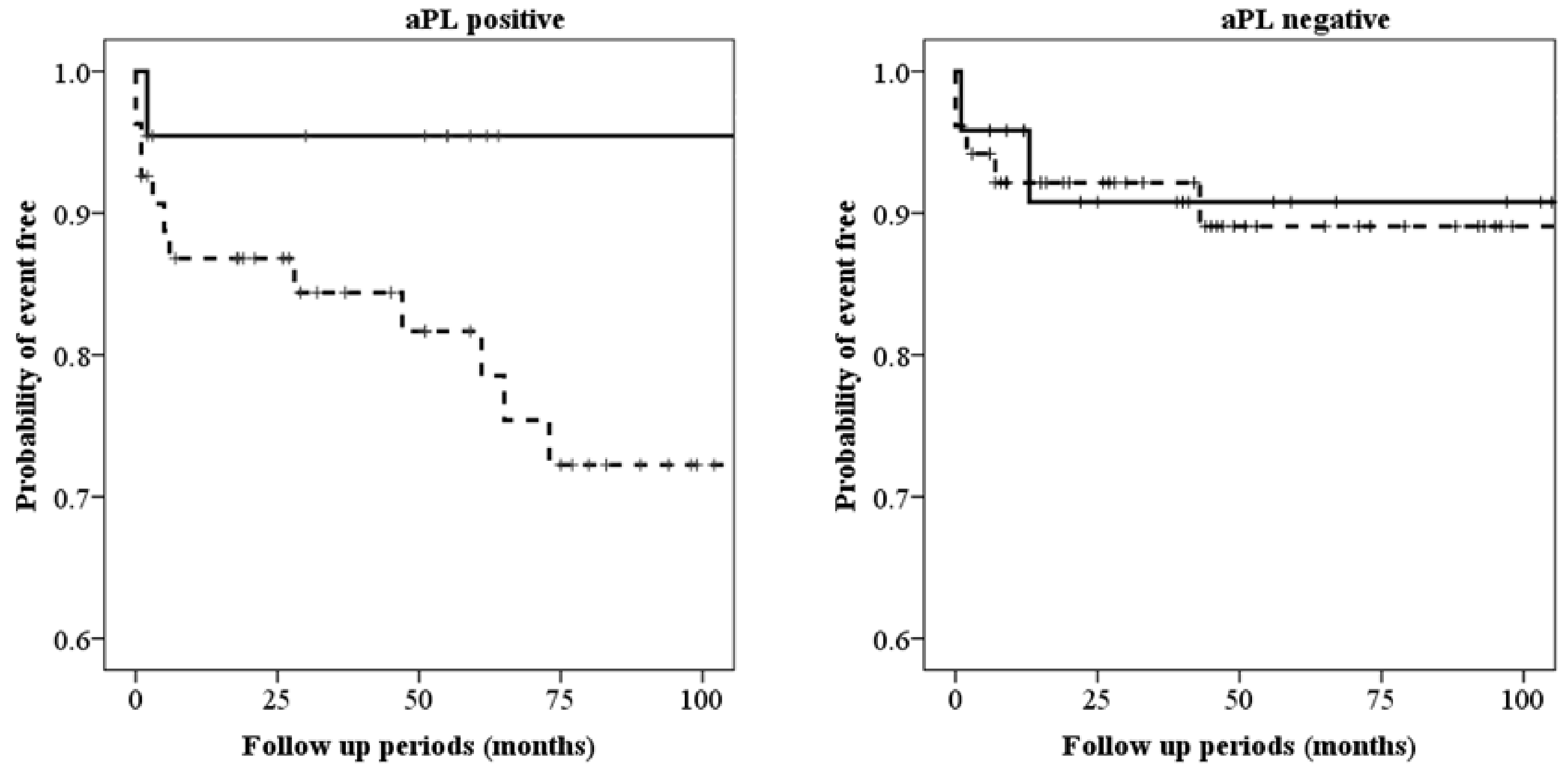